\title{
Stochastic and deterministic processes interact in the assembly of desert microbial communities on a global scale
}

\author{
Tancredi Caruso ${ }^{1,4}$, Yuki Chan ${ }^{2,4}$, Donnabella C Lacap ${ }^{2,4}$, Maggie CY Lau ${ }^{2}$, \\ Christopher P McKay ${ }^{3}$ and Stephen B Pointing ${ }^{2}$ \\ ${ }^{1}$ Institut für Biologie, Plant Ecology, Freie Universität Berlin, Berlin, Germany; ${ }^{2}$ School of Biological Sciences, \\ The University of Hong Kong, Hong Kong, China and ${ }^{3}$ NASA Ames Research Center, Moffet Field, Mountain \\ View, CA, USA
}

\begin{abstract}
Extreme arid regions in the worlds' major deserts are typified by quartz pavement terrain. Cryptic hypolithic communities colonize the ventral surface of quartz rocks and this habitat is characterized by a relative lack of environmental and trophic complexity. Combined with readily identifiable major environmental stressors this provides a tractable model system for determining the relative role of stochastic and deterministic drivers in community assembly. Through analyzing an original, worldwide data set of 16S rRNA-gene defined bacterial communities from the most extreme deserts on the Earth, we show that functional assemblages within the communities were subject to different assembly influences. Null models applied to the photosynthetic assemblage revealed that stochastic processes exerted most effect on the assemblage, although the level of community dissimilarity varied between continents in a manner not always consistent with neutral models. The heterotrophic assemblages displayed signatures of niche processes across four continents, whereas in other cases they conformed to neutral predictions. Importantly, for continents where neutrality was either rejected or accepted, assembly drivers differed between the two functional groups. This study demonstrates that multi-trophic microbial systems may not be fully described by a single set of niche or neutral assembly rules and that stochasticity is likely a major determinant of such systems, with significant variation in the influence of these determinants on a global scale. The ISME Journal (2011) 5, 1406-1413; doi:10.1038/ismej.2011.21; published online 3 March 2011
\end{abstract}

Subject Category: microbial population and community ecology

Keywords: desert; bacteria; hypolith; niche; neutrality; global scale

\section{Introduction}

Neutral theories predict that random patterns in species co-occurrence and environmentally independent spatial autocorrelation (for example, dispersal) should be the main features of community structure if demographic stochasticity and limited dispersal alone were driving population dynamics (Chesson and Huntly, 1989; Engen and Lande, 1996; Bell, 2000; Hubbell, 2001; Whitfield, 2002; McGill, 2003; Volkov et al., 2003, 2004; Chave, 2004; Connell et al., 2004; Maurer and McGill, 2004; Bell et al., 2005; Sloan et al., 2006; Bell, 2010). Conversely, more deterministic processes driven by species interaction and niche partitioning are predicted to produce segrega-

Correspondence: T Caruso, Institut für Biologie, Plant Ecology, Freie Universität Berlin, Altensteinstr. 6, Berlin 14195, Germany or SB Pointing, School of Biological Sciences, The University of Hong Kong, Hong Kong, China.

E-mail: tancredi.caruso@fu-berlin.de or pointing.steve@gmail. com

${ }^{4}$ These authors contributed equally to this work.

Received 9 August 2010; revised 28 January 2011; accepted 1 February 2011; published online 3 March 2011 tion in terms of species co-occurrence or even aggregation (Chave, 2004; Dornelas et al., 2006; Chase, 2007), if niche partitioning interacts with environmental stochasticity (that is, disturbance regime) or with extreme changes in key abiotic variables such as water availability and temperature. Theories have been tested against communities with a single trophic level including microorganisms (Volkov et al., 2003, 2004; Dumbrell et al., 2010; Ofiteru et al., 2010) and recently by manipulating relatively simple systems such as aquatic bacterial communities (Bell, 2010). Community assembly is highly dependent on a multitude of bottom-up and top-down trophic influences, which depend upon a biological diversity encompassing multiple phyla and realms. As theories based either on neutrality or competition should be strictly applied to a single trophic level (for example, Hubbell, 2001; Tilman, 2004), testing such theories by means of observational evidence is challenging in that other factors such as trophic interactions are actually operating in the field. A simple multi-trophic model system has not previously been examined with respect to elucidating the relative role of stochastic and 
deterministic determinants of microbial community structure using molecular ecological approaches.

Here, we analyze an original, worldwide data set obtained from the most extreme deserts across every continent on the Earth (Figure 1). We targeted the hypolithic niche, ventral surfaces of quartz rocks embedded in desert soils, as this substrate is a ubiquitous feature of arid regions (Thomas, 1997) and a relatively inert substrate that supports development of microbial communities that are distinct from surrounding soils (Pointing et al., 2009). Hypolithic communities are thought to develop as a result of marginal gains in niche favorability in terms of moisture availability and possible thermal buffering (Schlesinger et al., 2003; Warren-Rhodes et al., 2006; Tracy et al., 2010), although micro-niche variability may occur between individual rocks (Tracy et al., 2010). Several studies have indicated that hypolithic communities comprise relatively simple communities dominated by cyanobacteria, with a heterotrophic component largely represented by the Acidobacteria, Actinobacteria, Bacteriodetes and proteobacterial phyla (Schlesinger et al., 2003; Warren-Rhodes et al., 2006, 2007; Pointing et al., 2007, 2009; Wood et al., 2008; Wong et al., 2010). These studies have indicated that the Archaea and eukaryal microorganisms are either absent or present with low abundance. The lithic communities formed by bacteria in hyperarid deserts thus provide ecologists with a tractable global-scale model system for testing theories of community assembly, comprising relatively few phyla that are readily identified as producers or consumers (Warren-Rhodes et al., 2006; Pointing et al., 2007, 2009) Therefore, it offers the opportunity to analyze a natural community with a very limited (basically two) number of trophic levels and without the potentially confounding effect of higher taxa such as protistans and metazoans. This allowed us to focus on both the entire community and/or part of it.

The global distribution of hyperarid deserts allowed us to disentangle the contribution of three main factors: climatic region (based upon long-term mean annual temperature and precipitation), geographic region and continent, and spatial autocorrelation as described by patterns of community dissimilarities accounted for by the geographic position of each sample, that is, after partialling out (Legendre and Legendre, 1998; Cottenie, 2005) the effect of climatic region and continent. However, as variance partitioning itself may not sufficiently disentangle the signature of different assembly processes (see the recent critique by Smith and Lundholm, 2010) we further conducted analysis with null and neutral models, which were used to test null hypotheses framed in terms of species cooccurrence and level of community dissimilarity expected under neutrality (Gotelli, 2000; Dornelas et al., 2006; Etienne, 2007; Dornelas, 2010; Gotelli et al., 2010).

\section{Materials and methods}

Environmental DNA was recovered from the total hypolithic bacterial biomass on quartz pebbles from

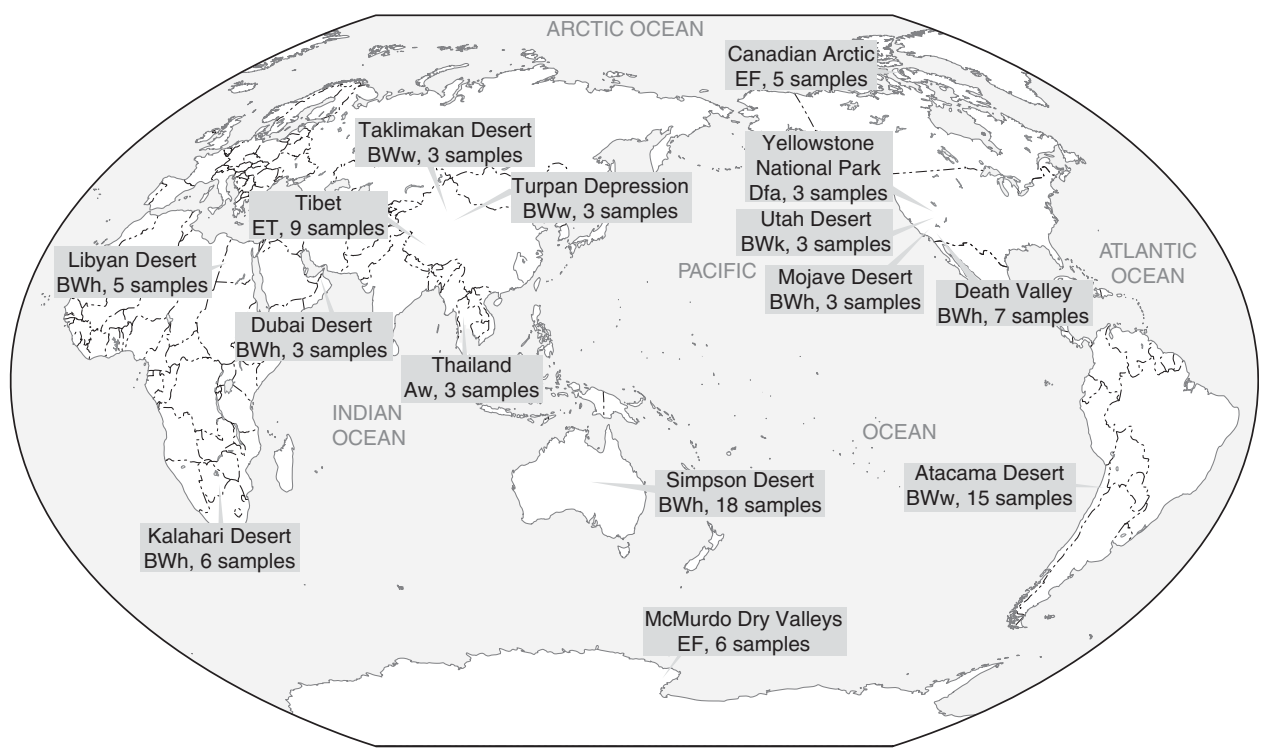

Figure 1 World map showing locations of desert sampling sites and number of samples recovered per location. Two non-arid locations (Chiang Mai, Thailand (warm tropical) and Yellowstone National Park, USA (cold temperate)) were also included for reference, but were not included in the analysis. Climate classification follows an updated Köppen-Geiger scheme (Peel et al., 2007) as follows, where $\mathrm{MAT}=$ mean annual temperature $\left({ }^{\circ} \mathrm{C}\right)$ and $\mathrm{MAP}=$ mean annual precipitation $\left(\mathrm{xP}_{\text {threshold }}\right)$ : BWh, hot arid desert $(\mathrm{MAT}>18, \mathrm{MAP}<5)$; BWk, cold arid desert (MAT $<18$, MAP $<5$ ); ET, polar tundra (MAT $<10)$; EF, polar frost (MAT $<0$ ); Dfa, cold winter $($ MAT hot $>10$, $\left.\mathrm{MAT}_{\text {cold }}<0\right)$. Deserts experiencing mean annual temperature $<18{ }^{\circ} \mathrm{C}$ but hot diurnal and/or seasonal temperatures (Atacama, Taklimakan, Turpan) are categorized as warm arid deserts (BWw; MAT>10, MAP <5; Thomas, 1997). Quartz substrate was identified using USGS (The United States Geological Survey) mineral identification criteria (http://pasadena.wr.usgs.gov/office/given/geo1/). 
desert locations worldwide, for a minimum of three rocks per sampling location $(N=92$; Figure 1$)$. Two non-arid locations (Chiang Mai, Thailand (warm tropical) and Yellowstone National Park, USA (cold temperate)) were also included for reference but were not included in the analysis. PCR amplification of $16 \mathrm{~S}$ rRNA genes was carried out using universal bacteria primers 8F-1391R (Lane, 1991). Restriction digests (MspI) of FAM-labelled PCR amplicons were subjected to fragment analysis (terminal restriction fragment length polymorphism) by capillary electrophoresis (Applied Biosystems 3730 Genetic Analyzer, Foster City, CA, USA). The software Perl and R were used to identify true peaks and bin fragments of similar size (Abdo et al., 2006). Peak intensity was used as a proxy for relative abundance. Analysis of Bray Curtis similarities was used to identify clusters of similar terminal restriction fragment length polymorphism profiles and from these seven clone libraries (each library $n=102-111$, total $n=726$ clones) were generated. Each pooled sample was gel-purified and used as template for construction of the clone libraries (Qiagen PCR Cloning ${ }^{\text {plus }}$ kit, Qiagen, Valencia, CA, USA). Plasmids were extracted from positive transformants (Mini-M Plasmid DNA extraction system, Viogene, Taipei, Taiwan) and screened by restriction fragment length polymorphism using the restriction endonucleases $M s p I$ and $C f o I$. At least three samples from each distinct restriction fragment length polymorphism pattern were sequenced using the BigDye Terminator Cycle Sequencing kit (Applied Biosystems; Applied Biosystems 3730 Genetic Analyzer). A sequence similarity cut-off value of $97 \%$ was used to delineate operational taxonomic unit by DOTUR (Schloss and Handelsman 2005). Approximate phylogenetic affiliations were determined by BLAST searches against the NCBI GenBank database (http://www.ncbi.nlm.nic.gov/). Sequences were then subjected to virtual digestion and matched to terminal restriction fragment length polymorphism peaks, which allowed identification to at least phylum level for $92 \%$ of peaks. Phylotypes were categorized as producers (phototrophs) only if they affiliated with the photoautotrophic cyanobacteria, photoheterotrophic phyla such as the
Chloroflexi were excluded. No phylogenetic signatures for chemoautotrophic bacteria were detected. Other phylotypes were all classified as heterotrophs. This follows the rationale that high taxonomic ranks maintain physiological relevance for these phyla (Philippot et al., 2010).

We quantified the relative importance of the environment (accounted for by the climatic region within which the desert was classified (Peel et al., 2007), the biogeographical effect due to the identity of the continent to which the deserts belonged and spatial processes (accounted for by the effect of the geographic position of every single sample) by decomposing the total variation in the community matrix into unique environmental, continental and spatial components with corresponding $P$ values using partial-correspondence and redundancy analysis (Legendre and Legendre, 1998; Cottenie, 2005; online Supplementary Information). Null model analysis of species co-occurrence was performed following Gotelli (2000) and Gotelli and Entsminger (2001); details are given in the online Supplementary Information. Using the species abundance vector available for each sample (that we defined as the local community), we estimated the neutral diversity $(\theta)$ and immigration $(I)$ parameters using the neutral sampling formula for multiple samples by Etienne (2007) for each continent. Using the maximum likelihood estimates of $\theta$ and $I$, we created a number of local communities corresponding to the size of the original data sets. Using the Jaccard's index (results were not affected by the specific index used) we quantified community dissimilarities in terms of species composition for both the actual and simulated data sets and we compared them with a $t$-statistics based on bootstrap procedures (Efron and Tibshirani, 1993).

\section{Results and Discussion}

Sequence-based identification of globally dispersed hypolithic communities revealed that all were dominated by cyanobacteria, the primary producers of the system, whereas heterotrophic bacteria had much lower abundances and very scattered distribu-

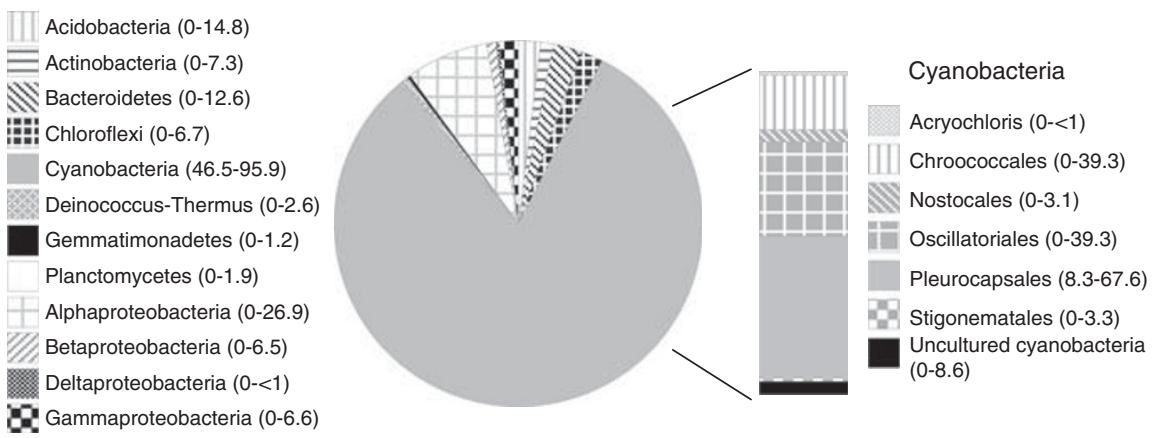

Figure 2 Summary of desert hypolithic community structure, based upon environmental 16S rRNA gene sequences. Bracketed numbers show the range for phyla among all samples $(N=92)$. All values shown are percentage of overall community. 
Table 1 Decomposition of the total variation in community matrix into unique environmental (climate) and spatial (continent effect and geographic position) components using (partial) redundancy analysis

\begin{tabular}{|c|c|c|c|c|c|c|}
\hline \multirow{2}{*}{$\begin{array}{l}\text { Assemblage } \\
\text { Source }\end{array}$} & \multicolumn{2}{|c|}{ Heterotrophs+phototrophs } & \multicolumn{2}{|c|}{ Heterotrophs } & \multicolumn{2}{|c|}{ Phototrophs } \\
\hline & Variance (\%) & $\mathrm{P}$-value & Variance (\%) & $\mathrm{P}$-value & Variance (\%) & $\mathrm{P}$-value \\
\hline Climate category & $<0.1$ & 0.05 & $<0.1$ & NS & $<0.1$ & NS \\
\hline PCNMs $^{\mathrm{a}}$ (spatial patterns) & 5 & $<0.01$ & 4 & 0.05 & 7 & $<0.01$ \\
\hline Continent & 5 & $<0.01$ & 3 & NS & $<0.1$ & NS \\
\hline Spatial structured climate and continent effect & 12 & NT & 21 & NT & 20 & NT \\
\hline Explained & 22 & & 28 & & 27 & \\
\hline Unexplained & 78 & & 72 & & 73 & \\
\hline
\end{tabular}

Abbreviations: NS, not significant; NT, not testable.

${ }^{a}$ Eigenvectors from the principal coordinate analysis of the neighbor matrix (PCNM) based on great circle distance between samples, which was calculated by finding the interior spherical angle between the two points using the Haversine Formula (Sinnott, 1984) and then multiplying that angle by the radius of the Earth.

tion (Figure 2). In some individual samples, heterotrophs dominated the assemblage at the smallest scale of the analysis (that is, an individual rock, the smallest sampling unit). At the whole community level, the climatic region and the identity of the continent to which the deserts belonged together with the geographical position of each sample accounted for $22 \%$ of overall data variance (Table 1 ). Most of this variance was accounted for by the spatially structured effect of the continent and climate. However, although there was no effect of climate after accounting for the effect of continent and spatial patterns, there was a significant effect of the continent after accounting for spatial patterns and climate, suggesting the existence of biogeographical clustering at a global scale (Figure 3a). Furthermore, there was a significant effect of spatial patterns independent of climate and continent, which suggests the existence of climate independent spatial patterns that are internal to continents, and also among deserts within continents.

We thus re-analyzed the total variation within the community matrix by examining the photosynthetic and heterotrophic assemblages separately. The effect of climate or continent alone was no longer detectable, whereas the effect of spatial patterns after accounting for climate and continent remained marginally (heterotrophs) or highly (phototrophs) significant. Overall, the pure spatial component together with the spatially structured effect of climate and continent accounted for most (>75\%) of the so-called 'explained' variance (Table 1). Qualitatively, the decreased importance of the continent effect after splitting the community matrix into the two functional components (photo- and heterotrophs) is highlighted by the fact that the geographical clustering observed for the entire community was much less clear when the analysis included photosynthetic or heterotrophic species only (Figures $3 \mathrm{~b}$ and c). Overall about $70-80 \%$ of variance remained unexplained. Certainly, this is partly due to the fact that we introduced the environmental effect using a relatively broad approximation (climate categories based upon long-term mean annual temperature and precipitation), which ignored any unmeasured local environmental drivers that may be involved in determining community structure (for example, see Tracy et al., 2010). However, given previous studies on these communities that showed hypoliths develop independently of the surrounding soil (Warren-Rhodes et al., 2006; Pointing et al., 2007, 2009; Tracy et al., 2010 ) owing to the specific microclimatic conditions of rocks, and that climate and continent were independent of spatial patterns observed, it is likely that a large amount of this variation is due to environmental (for example, see Dornelas et al., 2006; Pointing et al., 2009) and/or demographic stochasticity (sensu Hubbell, 2001 and Tilman, 2004).

In order to test this hypothesis, we performed a null model analysis (Gotelli, 2000; Gotelli and Entsminger, 2001) to test whether patterns of species co-occurrence (measured by the C-score, the results of which overlapped with the other indices proposed by Gotelli and Entsminger, 2001) were distinguishable from random patterns (Table 2). When we focused on the photosynthetic assemblage alone, we observed neither significant segregation nor aggregation. On the contrary, heterotrophs were significantly segregated (species co-occurred less frequently than randomly predicted) in four continents. It was evident from comparing the different tested assemblages (Table 2) that the detection of non-random patterns by null model analysis on the entire community was mainly driven by the heterotrophic communities. However, in the case of Africa non-random pattern in species co-occurrence were detected when the entire community was analyzed, whereas non-random patterns were not detected when the two groups were analyzed separately. This result suggests that species segregation is potentially driven by interactions between the two groups or by a common response of the two groups to the environment, which may reinforce the power to detect a non-random signal at the level of the entire bacterial community. 

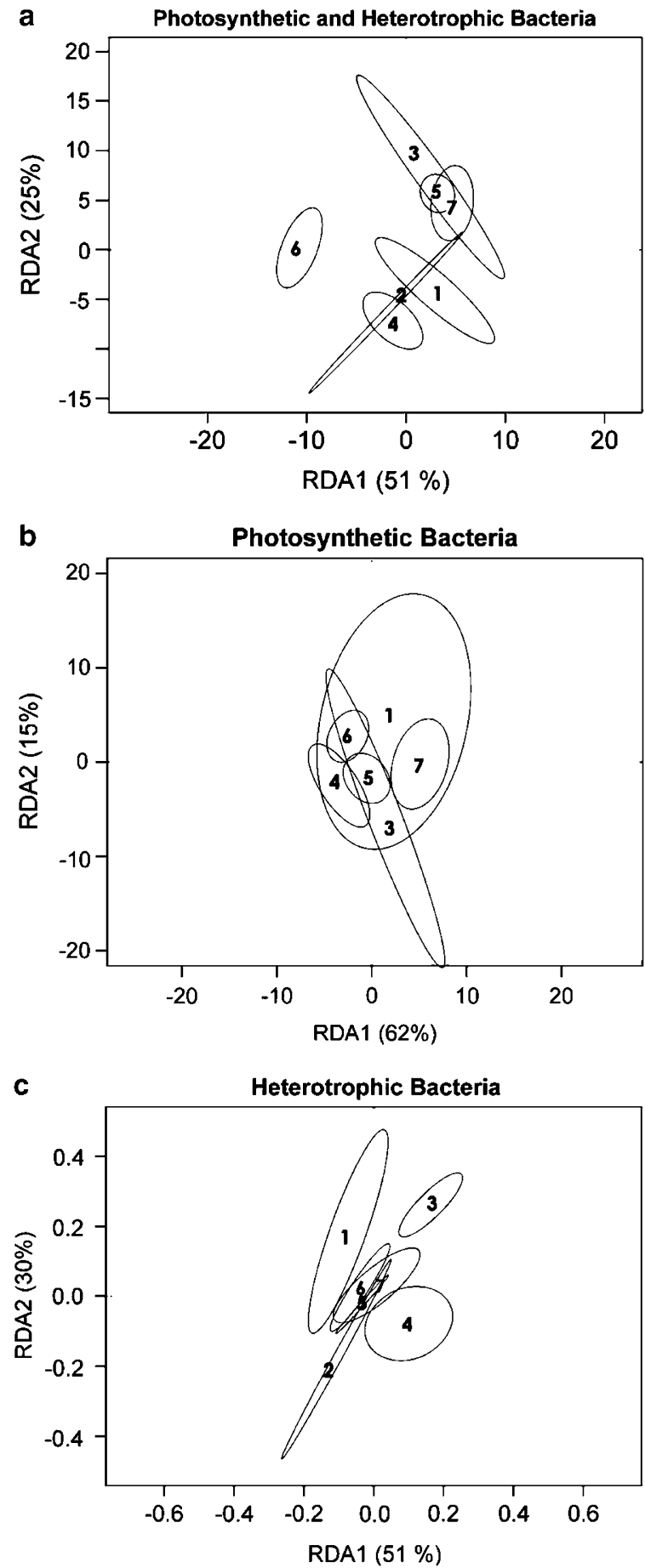

Figure 3 Ordination plots of the redundancy analysis on the bacterial community matrix as dependent on continental location of each desert. In this case, the analysis represents the unique contribution of continent identity to (a) the entire bacterial community; (b) phototrophic bacteria; and (c) heterotrophic bacteria. The effect of continent was significant for the entire community only. Instead of plotting each sampling point, ellipses were drawn to represent the standard error around the continental centroids: 1, Africa; 2, Antarctica; 3, Arctic; 4, Asia; 5, Australia; 6, North America; 7, South America.

Given that patterns in species co-occurrence of the photosynthetic assemblage and three heterotrophic assemblages were indistinguishable from a random pattern, we tested the prediction of the neutral model, that is, that patterns in species distribution are consistent with community assembly rules that postulate demographic stochasticity and limited dispersal are the only drivers of population dynamics and that species are ecologically equivalent in terms of niche optima. We estimated neutral diversity and dispersal parameters using Etienne's formula for multiple samples (Etienne, 2007) and we used this estimate for simulating neutral communities with same size, diversity and dispersal limitation of our photosynthetic assemblages. We then calculated community dissimilarity for the actual and simulated data sets and compared them in order to test the null hypothesis that there is no significant shift in the mean level of dissimilarity (Dornelas et al., 2006; Etienne, 2007). Results were highly dependent on the respective groups (Table 3). For the phototrophs, in four cases (Australia, Arctic, South America and North America) our findings rejected the neutral model. However, the Arctic community diverged with dissimilarity higher than the predicted neutral community, which suggests a major role for environmental stochasticity mediated by niche processes (Dornelas et al., 2006). In the other three cases, dissimilarities converged (that is, lower than expected), which has been proposed to be a signature of niche processes as convergence implies that local communities, with all other conditions being equal and tended to a predictable composition, should depend on the output of nichemediated competition (Tilman, 2004; Etienne, 2007; see also Sloan et al., 2006 for examples on bacterial community). In the remaining cases, data were consistent with the neutral models. This suggests that population dynamics can be approximated by stochastic demography and limited dispersal, though there is no direct implication that these mechanisms were the only forces shaping community assembly. For heterotrophs, three cases rejected the neutral model and dissimilarities were lower than prediction, whereas the remaining cases were consistent with the neutral model. It is important to note that neutral theory was rejected for both functional groups in African community, whereas others accepted neutrality for either one of the functional groups (Table 3). For example, in Asia, neutrality was rejected for the heterotrophs, but accepted for the phototrophs although the converse applied to Arctic communities.

\section{Conclusion}

Overall, our analysis demonstrates that for our global desert model system, patterns of community structure depend on a complex balance between deterministic and stochastic forces acting on populations at different spatial scales and within different functional groups. This extends the observation that events such as drought or disturbance 
Table 2 Results of testing whether species co-occurrence in bacterial community can be approximated by random distributions generated by statistical models (null model analysis)

\begin{tabular}{|c|c|c|c|c|c|c|}
\hline \multirow[t]{2}{*}{ Continent } & \multicolumn{2}{|c|}{ Phototrophs } & \multicolumn{2}{|c|}{ Heterotrophs } & \multicolumn{2}{|c|}{ Phototrophs+heterotrophs } \\
\hline & Effect size & $\mathrm{P}$-value & Effect size & $\mathrm{P}$-value & Effect size & $\mathrm{P}$-value \\
\hline Africa & 1.15 & 0.14 & 1.15 & 0.18 & 3.61 & $<0.01$ \\
\hline Arctic & 0.75 & 0.28 & 1.61 & 0.21 & -0.26 & 0.53 \\
\hline Antarctica & -0.38 & 0.87 & 1.23 & 0.39 & 1.80 & 0.20 \\
\hline Asia & -1.27 & 0.1 & 1.89 & 0.04 & 2.26 & 0.02 \\
\hline South America & 1.87 & 0.05 & 2.67 & 0.01 & 5.8 & $<\mathbf{0 . 0 1}$ \\
\hline North America & 2.11 & 0.05 & 2.12 & 0.03 & 3.59 & $<\mathbf{0 . 0 1}$ \\
\hline Australia & 1.24 & 0.12 & 2.28 & 0.02 & 3.75 & $<\mathbf{0 . 0 0 1}$ \\
\hline
\end{tabular}

All $P$-values are tail probabilities of observed C-score obtained by random permutations. Those in bold indicates the rejection of the null hypothesis at $P \leqslant 0.05$. The null hypothesis is that species co-occur neither more (negative effect size) nor less (positive effect size) frequently than expected under a random distribution. The C-score measures the extent by which species are not co-occurring. The effect size scales the difference between the actual and simulated C-scores in units of standard deviations, which allows comparison among different data sets.

Table 3 Results of testing whether bacterial community can be approximated by the processes postulated by neutral models (demographic drift and limited dispersal with parameters estimated using Etienne's sampling formula)

\begin{tabular}{lccccc}
\hline \multirow{2}{*}{ Continent } & \multicolumn{2}{c}{ Phototrophs } & & \multicolumn{2}{c}{ Heterotrophs } \\
\cline { 2 - 3 } \cline { 5 - 6 } & Effect size & $\mathrm{P}$ & & Effect size & $\mathrm{P}$ \\
\hline Africa & 0.8 & 0.45 & & -0.1 & 0.94 \\
Arctic & 2.6 & $<\mathbf{0 . 0 1}$ & & -0.6 & 0.53 \\
Asia & 1.6 & 0.11 & & -4.7 & $<\mathbf{0 . 0 0 1}$ \\
South America & -2.0 & $\mathbf{0 . 0 5}$ & & -1.2 & 0.23 \\
North America & -2.2 & $\mathbf{0 . 0 3}$ & & -3.1 & $<\mathbf{0 . 0 1}$ \\
Australia & -5.2 & $<\mathbf{0 . 0 0 1}$ & -0.4 & 0.7 \\
Antarctica & & $\mathrm{NT}$ & & -2.3 & $\mathbf{0 . 0 2}$ \\
& & & & &
\end{tabular}

Abbreviation: NT, not testable.

$P$-values represent the probability of bootstrapped $t$-test. Those in bold indicate the rejection of the null hypothesis at $P \leqslant 0.05$. For the neutral model, the null hypothesis is that observed mean dissimilarities between any two samples (Jaccard index) are the same as predicted under ecological neutrality. Positive effect size indicates that dissimilarity is higher than predicted (divergence), whereas negative effect size indicates the opposite (convergence). Phototrophic bacteria in Antarctica were too sparse and rare for a meaningful estimate of neutral parameters. The effect size scales the mean dissimilarity between the actual and simulated communities in units of standard deviations, which allows comparison among different data sets.

enhance niche-selection processes (Chase, 2007; Ofiteru et al., 2010). The important novelty is that a key component of the equation appears to be the differences in patterns of community structure for the functional groups that form the system (phototrophs and heterotrophs). This is particularly interesting in deserts and very extreme systems, where the role of niche partitioning at fine scales and biological interaction may generally have been underestimated, as it has previously been assumed that the extreme harshness of climate is the main if not the only determinant of species distribution (for example, see Friedmann and Galun, 1974). An implicit consequence of the classical view is that extremophilic communities could be expected to converge toward an average stable and low diversity community. Despite this, recent molecular evidence has indicated that hypolithic communities may harbor significant phylogenetic diversity among bacterial phyla (Warren-Rhodes et al., 2006; Pointing et al., 2007, 2009; Bahl et al., 2011).

Our data demonstrate that patterns generally interpreted as indicative of the presence of niche partitioning and non-neutral processes (for example, convergence due to relatively homogeneous environments) vary on a global biogeographical scale (continent) and also between phototrophic and heterotrophic bacteria within a given community. Given that the commonly encountered phyla in hypolithic communities include known desiccationtolerant taxa, this implies that most populations may withstand the high level of demographic stochasticity due to the patchiness of the environment in hyperarid deserts (Pointing et al., 2007, 2009; Warren-Rhodes et al., 2007; Tracy et al., 2010). Further, they may opportunistically react to the environmental stochasticity determined by rapid and extreme changes in temperature and water availability. Our data indicate that patterns of community structure resulting from these interactions may extend to inter-specific competition or indirect interaction. We hypothesize that these interactions may explain why the two functional groups generally displayed different assembly patterns. The case of Africa is particularly interesting, as both the null and neutral model failed to detect the signature of deterministic processes when phototrophs and heterotrophs were analyzed separately, whereas species co-occurrence was non-random when we analyzed the groups together. Although in several cases our data clearly demonstrated the signature of non-random and non-neutral processes at a very broad scale, relatively high levels of stochasticity remain the most striking feature of these communities. In fact, several functional assemblages could be approximated by the neutral model and the diverged phototrophs in the Arctic samples could reflect a strong influence due to 
environmental stochastic effect, which may cause increased levels of community dissimilarity (for example, see Dornelas et al., 2006).

Classical population models predict populations that closely track environmental stochasticity are highly prone to extinction (for example, see May, 1976), and communities that are dominated by species characterized by the type of population dynamics we identified in this study may be assumed to be highly susceptible to disturbance due to the environmental uncertainty that is expected with accelerated rates of climate change. These rates are of particular concern in arid systems, most notably those in polar regions (for example, see Steig et al., 2009), and thus it is timely to further unravel the mechanisms that drive community structure through the interaction of stochastic and deterministic processes.

\section{Conflict of interest}

The authors declare no conflict of interest.

\section{Acknowledgements}

This research was supported by the United States National Science Foundation, NASA Astrobiology Science and Technology for Exploring Planets (ASTEP) Programme, and the Hong Kong Research Grants Council (HKU7733/ $08 \mathrm{M}$ and HKU7763/10). T. Caruso was supported by EBESA IPY project $n^{\circ} 452$, SCAR EBA Programs, the Italian PNRA (Programma Nazionale di Ricerche in Antartide), Freie Universität Berlin and the Humboldt Foundation. The authors thank Professor Yuwadee Peerapornpisal (Chiang Mai University, Thailand) for providing tropical hypolith samples. We thank Jeff $\mathrm{R}$ Powell for commenting on the manuscript.

\section{References}

Abdo Z, Schuette UME, Bent SJ, Williams CJ, Forney LJ, Joyce P. (2006). Statistical method for characterizing diversity of microbial communities by analysis of terminal restriction fragment length polymorphisms of 16S rRNA genes. Environ Microbiol 8: 929-938.

Bahl J, Lau MCY, Dhanesakaran V, Smith GJD, Cary C, Lee CK et al. (2011). Ancient origins determine global biogeography of hot and cold desert cyanobacteria. Nature Commun 2: 163.

Bell G. (2000). The distribution of abundance in neutral communities. Am Nat 155: 606-617.

Bell T. (2010). Experimental tests of the bacterial distancedecay relationship. ISME J 4: 1357-1365.

Bell T, Newman JA, Silverman BW, Turner SL, Lilley AK. (2005). The contribution of species richness and composition to bacterial services. Nature 436: 1157-1160.

Chase JM. (2007). Drought mediates the importance of stochastic community assembly. Proc Natl Acad Sci USA 104: 17430-17434.
Chave J. (2004). Neutral theory and community ecology. Ecol Lett 7: 241-253.

Chesson P, Huntly N. (1989). Short-term instabilities and long-term community dynamics. Trends Ecol Evol 4: 293-298.

Connell JH, Hughes TP, Wallace CC, Tanner JE, Harms KE, Kerr AM. (2004). A long term study of competition and diversity of corals. Ecol Monogr 74: 179-210.

Cottenie K. (2005). Integrating environmental and spatial processes in ecological community dynamics. Ecol Lett 8: 1175-1182.

Dornelas M. (2010). Disturbance and change in biodiversity. Phil Trans $R$ Soc B 365: 3719-3727.

Dornelas M, Connolly SR, Hughes TP. (2006). Coral reef diversity refutes neutral theory. Nature 440: 80-82.

Dumbrell AJ, Nelson M, Helgason T, Dytham C, Fitter AH. (2010). Relative role of niche and neutral processes in structuring a soil microbial community. ISME J 4: $337-345$.

Efron B, Tibshirani R. (1993). An Introduction to the Bootstrap. Chapman and Hall: New York, USA.

Engen S, Lande R. (1996). Population dynamic models generating the lognormal species abundance distribution. Math Biosci 132: 169-183.

Etienne RS. (2007). A neutral sampling formula for multiple samples and an exact test of neutrality. Ecol Lett 10: 608-618.

Friedmann I, Galun M. (1974). Desert algae, lichens and fungi. Desert Biology, vol. II. Academic Press: New York, USA.

Gotelli NJ. (2000). Null model analysis of species co-occurrence patterns. Ecology 81: 2606-2621.

Gotelli NJ, Entsminger GL. (2001). Swap and fill algorithms in null model analysis: rethinking the Knight's Tour. Oecologia 129: 281-291.

Gotelli NJ, Graves GR, Rahbek C. (2010). Macroecological signals of species interactions in the Danish avifauna. Proc Natl Acad Sci USA 107: 5030-5035.

Hubbell SP. (2001). The Unified Neutral Theory of Biodiversity and Biogeography. Princeton Univ. Press: Princeton.

Lane DJ. (1991). 16S/23S rRNA sequencing. In: Stackebrandt E, Goodfellow M (eds). Nucleic Acid Techniques in Bacterial Systematics. John Wiley \& Sons: Chichester, UK, pp 115-148.

Legendre P, Legendre L. (1998). Numerical Ecology. Elsevier: Amsterdam.

Maurer BA, McGill BJ. (2004). Neutral and non-neutral macroecology. Basic Appl Ecol 5: 413-422.

May RM. (1976). Models for single populations. In: May RM (ed.). Theoretical ecology: Principles and Applications. Blackwell Scientific Publications: Oxford, UK.

McGill BJ. (2003). Strong and weak tests of macroecological theory. Oikos 102: 679-685.

Ofiteru ID, Lunn M, Curtis TP, Wells GF, Criddle CS, Francis CA et al. (2010). Combined niche and neutral effects in a microbial wastewater treatment community. Proc Natl Acad Sci USA 107: 15345-15350.

Peel MC, Finlayson BL, McMahon TA. (2007). Updated world map of the Köppen-Geiger climate classification. Hydrol Earth Syst Sci 11: 1633-1644.

Philippot L, Andersson SGE, Battin TG, Prosser JI, Schimel JP, Whitman WB et al. (2010). The ecological coherence of high bacterial taxonomic ranks. Nat Rev Microbiol 8: 523-529.

Pointing SB, Chan Y, Lacap DC, Lau MCY, Jurgens JA, Farrell RL. (2009). Highly specialized microbial 
diversity in hyper-arid polar desert. Proc Natl Acad Sci 106: 19964-19969.

Pointing SB, Warren-Rhodes K, Lacap DC, Rhodes KL, McKay CP. (2007). Hypolithic community shifts occur as a result of liquid water availability along environmental gradients in China's hot and cold hyperarid deserts. Environ Microbiol 9: 414-424.

Schlesinger WH, Pippin J, Wallenstein M, Hofmockel K, Klepeis D, Hahall B. (2003). Community composition and photosynthesis by photoautotrophs under quartz pebbles, southern Mojave Desert. Ecology 84: 3222-3231.

Schloss PD, Handelsman J. (2005). Introducing DOTUR, a computer program for defining operational taxonomic units and estimating species richness. Appl Environ Microbiol 71: 1501-1506.

Sinnott RW. (1984). Virtues of the Haversine. Sky and Telescope 68: 159.

Sloan WT, Mary Lunn M, Woodcock S, Head IM, Nee S, Curtis TP. (2006). Quantifying the roles of immigration and chance in shaping prokaryote community structure. Env Microbiol 8: 732-740.

Smith TW, Lundholm J. (2010). Variation partitioning as a tool to distinguish between niche and neutral processes. Ecography 33: 648-655.

Steig EJ, Schneider DP, Rutherford SD, Michael E, Mann ME, Comiso JC et al. (2009). Warming of the Antarctic ice-sheet surface since the 1957 International Geophysical Year. Nature 457: 459-462.

Thomas DSG. (1997). Arid zones: their nature and extent. In: Thomas DSG (ed.). Arid Zone Geomorphology, 2nd edn Wiley: Chichester, pp 3-12.
Tilman D. (2004). Niche tradeoffs, neutrality, and community structure: a stochastic theory of resource competition, invasion, and community assembly. Proc Natl Acad Sci USA 101: 10854-10861.

Tracy CR, Streten-Joyce C, Dalton R, Nussear KE, Gibb KS, Christian KA. (2010). Microclimate and limits of photosynthesis in a diverse community of hypolithic bacteria in northern Australia. Environ Microbiol 12: 592-607.

Volkov I, Banavar JR, Hubbell SP, Maritan A. (2003). Neutral theory and relative species abundance in ecology. Nature 424: 1035-1037.

Volkov I, Banavar JR, Maritan A, Hubbell SP. (2004). The stability of forest biodiversity. Nature 427: 696.

Warren-Rhodes KA, Rhodes KL, Boyle LN, Pointing SB, Chen Y, Liu S et al. (2007). Lithic cyanobacterial ecology across environmental gradients and spatial scales in China's hot and cold deserts. FEMS Microbiol Ecol 61: 470-482.

Warren-Rhodes KA, Rhodes KL, Pointing SB, Ewing SA, Lacap DC, Gómez-Silva B et al. (2006). Hypolithic cyanobacteria, dry limit of photosynthesis and microbial ecology in the hyperarid Atacama Desert, Chile. Microb Ecol 52: 389-398.

Whitfield J. (2002). Ecology: Neutrality versus the niche. Nature 417: 480-481.

Wong FKY, Lacap DC, Lau MCY, Aitchison JC, Cowan DA, Pointing SB. (2010). Hypolithic microbial community of quartz pavement in the high-altitude tundra of Central Tibet. Microb Ecol 60: 730-739.

Wood SA, Rueckert A, Cowan DA, Cary SC. (2008). Sources of edaphic cyanobacterial diversity in the Dry Valleys of Eastern Antarctica. ISME J 2: 308-320.

Supplementary Information accompanies the paper on The ISME Journal website (http://www.nature.com/ismej) 